\title{
Femtosecond fluorescence up-conversion spectroscopy of a rotation-restricted azobenzene after excitation to the $S_{1}$ state $\dagger$
}

\author{
T. Pancur, ${ }^{a}$ F. Renth, ${ }^{* a}$ F. Temps, ${ }^{* a}$ B. Harbaum, ${ }^{b}$ A. Krüger, ${ }^{b}$ R. Herges ${ }^{b}$ and \\ Chr. Näther ${ }^{c}$ \\ ${ }^{a}$ Institut für Physikalische Chemie, Christian-Albrechts-Universität zu Kiel, Ludewig-Meyn-Str. \\ 8,D-24098Kiel, Germany.E-mail: renth@phc.uni-kiel.de,temps@phc.uni-kiel.de \\ ${ }^{\boldsymbol{b}}$ Institut für Organische Chemie, Christian-Albrechts-Universität zu Kiel, Otto-Hahn-Platz 3, \\ D-24098 Kiel, Germany \\ ${ }^{c}$ Institut für Anorganische Chemie, Christian-Albrechts-Universität zu Kiel, \\ Otto-Hahn-Platz 6-7, D-24098 Kiel, Germany
}

Received 23rd December 2004, Accepted 24th March 2005

First published as an Advance Article on the web 11th April 2005

Femtosecond time-resolved fluorescence up-conversion spectroscopy has been used in a study of the photoinduced isomerization reactions of a rotation-restricted trans-azobenzene (trans-AB) derivative capped by a crown ether (1), a chemically similar open derivative (2), and unsubstituted trans-AB (3) after excitation to the $\mathrm{S}_{1}\left(\mathrm{n} \pi^{*}\right)$ state at $\lambda=475 \mathrm{~nm}$ in dioxane solution. The observed biexponential temporal fluorescence profiles for 1 and 2 were almost indistinguishable within experimental error. The fitted fast fluorescence decay times $( \pm 2 \sigma)$ for the two compounds were $\tau_{1}(\mathbf{1})=(0.79 \pm 0.20)$ and $\tau_{1}(\mathbf{2})=(1.05 \pm 0.20) \mathrm{ps}$, compared to $\tau_{1}(\mathbf{3})=(0.37 \pm 0.06) \mathrm{ps}$. The second decay components could be described with $\tau_{2}(\mathbf{1})=(20.3 \pm 9.5)$ resp. $\tau_{2}(\mathbf{2})=(19.0 \pm 6.0)$ ps, vs. $\tau_{2}$ $(3)=(3.26 \pm 0.85)$ ps. The very similar lifetimes strongly suggest that trans-cis isomerization of $\mathbf{1}$ and $\mathbf{2}$ after $\mathrm{S}_{1}$ excitation is governed by the same mechanism. Since 1 cannot isomerize by a simple large-amplitude rotation of one of the phenyl rings about the central $\mathrm{NN}$ bond, the isomerization dynamics of both ABs should be better described as "inversion" at the $\mathrm{N}$ atom(s) rather than large-amplitude "rotation".

\section{Introduction}

The photo-induced cis-trans isomerization of azobenzene (AB) is of substantial interest for optical data-storage devices ${ }^{1,2}$ or laser-triggered molecular switches. ${ }^{3-8}$ However, the detailed mechanisms and dynamics of this prototypical reaction are still a matter of controversy. A longstanding question is whether the isomerization takes place as "inversion" on one of the $\mathrm{N}$ atoms or as large-amplitude "rotation" about the central $\mathrm{NN}$ bond or whether it requires more complex nuclear motions. 9,10

Important experimental evidence pertaining to the ensuing reaction pathways has been reported by Rau and co-workers, who carried out quantum yield measurements for capped $A B$ derivatives, in which a large-amplitude rotational motion of the aromatic rings during isomerization should not be feasible. In particular, they studied the photoisomerization of an azobenzophane ${ }^{9}$ and the $\mathrm{AB}$ derivative $\mathbf{1}$, in which the two aromatic rings are connected by a crown ether. ${ }^{10}$ The experimental results were found to depend on whether the molecules were excited to the $S_{1}\left(n \pi^{*}\right)$ or to the $S_{2}\left(\pi \pi^{*}\right)$ electronic state. In particular, the quantum yields for isomerization of $\mathbf{1}$ and unsubstituted trans-AB (3) following excitation to the $S_{1}$ state were found to be practically the same within experimental error $(\Phi \approx 0.25$ and 0.29$)$, while substantial differences $(\Phi \approx 0.11$ and 0.29 , respectively) were encountered after excitation to the $\mathrm{S}_{2}$ state. ${ }^{10}$ Rau et al. thus concluded that $\mathrm{S}_{1}$ excitation leads to isomerization by inversion, which they assumed to take place

$\dagger$ Electronic supplementary information (ESI) available: Experimental details and crystal structure. See http://www.rsc.org/suppdata/cp/b4/ b419236b via a semi-linear planar CNNC transition state.
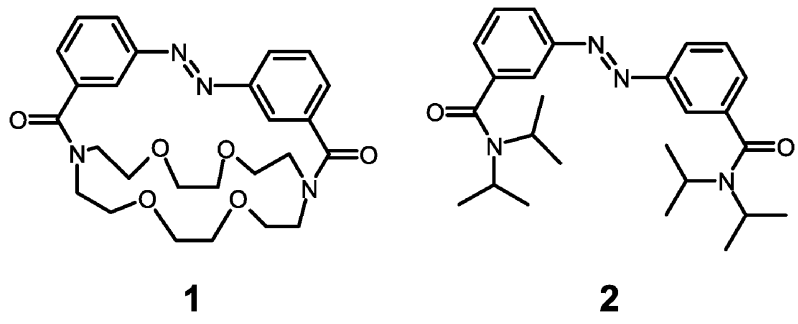

Transient absorption and fluorescence decay measurements carried out in the past few years showed that the isomerization of trans- $\mathrm{AB}$ after $\mathrm{S}_{1}$ excitation takes place on time scales between a few hundred femtoseconds and several picoseconds. ${ }^{11-16}$ The corresponding reaction of cis-AB has been found to be even faster. ${ }^{15,16}$ Most workers assumed the abovementioned semi-linear "inversion" pathway. ${ }^{1-13,15}$ However, the "rotational" isomerization route has recently received substantial support from a number of quantum chemical calculations. In particular, calculations on the semi-linear inversion mechanism in the $S_{1}$ state predicted a potential energy barrier for that pathway. ${ }^{14,17,18}$ In contrast, torsional motion has been suggested to lead to an easily accessible conical intersection $(\mathrm{CI})$ between the $\mathrm{S}_{1}$ and $\mathrm{S}_{0}$ states that can mediate an ultrafast radiationless relaxation to the ground state near the $90^{\circ}$ point of the CNNC out-of-plane rotation pathway. ${ }^{17-20}$ In addition, Diau recently proposed a "concerted inversion" channel. ${ }^{17}$ Chang et al. reported experimental evidence supporting the rotational isomerization route in low viscosity solvents (hexane) and the concerted inversion route in high viscosity solvents (ethylene glycol). ${ }^{21}$ A time-resolved investigation of the photochemical dynamics of the capped 
AB 1, in which the rotational isomerization pathway does not appear possible, thus seems to be of great interest.

In this publication, we report on first results of a femtosecond time-resolved experimental study of the dynamics of trans-1 (3,3'-bis(1,10-diaza-4,7,13,16-tetraoxa-18-crown-6-carbonyl)-trans-AB) and the chemically closely related open derivative trans-2 (3,3'-bis(diisopropylaminocarbonyl)-trans-AB) in dioxane solution following excitation to the $S_{1}$ state. 1 has previously been studied with femtosecond time resolution only after excitation to the $\mathrm{S}_{2}$ state at $\lambda=303 \mathrm{~nm},{ }^{13}$ while 2 has not been investigated at all to our knowledge. The molecules were excited to the $S_{1}$ state at $\lambda=475 \mathrm{~nm}$ and the ensuing dynamics were monitored by femtosecond fluorescence up-conversion spectroscopy. A comparison of the results for the two AB derivatives with each other and with data for the parent compound trans-AB (3) provides some interesting new insight into the ensuing isomerization mechanisms.

\section{Experimental section}

Experiments were carried out at room temperature employing a flow sample cell with $0.2 \mathrm{~mm}$ sapphire windows and $1 \mathrm{~mm}$ optical path length. ${ }^{16}$ Excitation light pulses at $\lambda=475 \mathrm{~nm}$ with $\approx 50 \mathrm{fs}$ (Gaussian fwhm) duration were provided by a home-built non-collinear optical parametric amplifier $(\text { NOPA })^{22}$ pumped by $\approx 200 \mu \mathrm{J}$ pulses from a regeneratively amplified Ti:sapphire femtosecond laser system (Clark MXR CPA 2001, $\lambda=775 \mathrm{~nm}, \approx 150 \mathrm{fs}$ fwhm, $1 \mathrm{kHz}$ repetition rate). Excitation pulses of approximately $0.5 \mu \mathrm{J}$ were focused into the sample cell to a spot size of $\approx 400 \mu \mathrm{m}$. Fluorescence was collected and refocused into a $0.2 \mathrm{~mm}$ BBO crystal (GWU) for up-conversion by type II sum frequency generation with the $775 \mathrm{~nm}$ gate pulses $(\approx 120 \mu \mathrm{J})$ from the Ti:Sa laser using a pair of off-axis parabolic mirrors (Melles-Griot, $f=119 \mathrm{~mm}$ ). A Schott OG550 filter between the mirrors removed scattered pump light. The upconverted light was bandpass filtered (Schott UG11), focused onto the entrance slit of an $f=0.1 \mathrm{~m}$ double monochromator (Jobin-Yvon HR 10), and detected with a photomultiplier (Hamamatsu R1527P) connected to a preamplifier (Stanford Research SR 445) and a gated photon counter (Stanford Research SR 400). Temporal fluorescence profiles were recorded by stepping the time delay of the gate pulses using a computer-controlled linear translation stage (Physik Instrumente M-126CG). The instrument response function (IRF), determined by upconverting scattered Raman light due to the $\mathrm{CH}$ stretching vibrations of the solvent or by cross correlation of the pump and gate pulses, was represented by a width parameter of $\tau_{\text {irf }} \approx 0.21$ ps (standard deviation of a normalized Gaussian, corresponding to a fwhm of $\approx 0.50 \mathrm{ps}$ ).

1 and 2 were synthesized as described in the electronic supplementary information (ESI) $\dagger$ following a modification of the procedure of Shinkai et al. ${ }^{23}$ Both compounds $(\approx 95$ and $97 \%$ purity, respectively) were checked by NMR spectroscopy. Compound 1 was additionally characterized by single crystal X-ray diffraction. $\dagger$ The structure of $\mathbf{1}$ which is depicted in Fig. 1 confirmed the trans configuration of the azo moiety. In addition, it showed that the two aromatic rings are slightly non-coplanar $\left(7^{\circ}\right)$ and the aminocarbonyl groups are twisted by $58^{\circ}$ with respect to the aromatic planes. The main impurity of 1 which could not be removed by simple means was the corresponding azoxybenzene. However, this molecule does not show any absorption at the excitation wavelength used $^{24}$ so that it does not affect the fluorescence measurements. Further details can be found in the ESI. $\dagger$

Sample solutions were prepared using dry dioxane (Merck, Uvasol) and pumped through the cell using a peristaltic pump (Ismatec, MS-CA 2/860, $6.8 \mathrm{ml} \mathrm{min}^{-1}$ flow rate). The number of experimental runs that could be made was limited by the small amounts of substance available. Measurements of the poorly soluble 1 were carried out at a concentration of $5 \times$

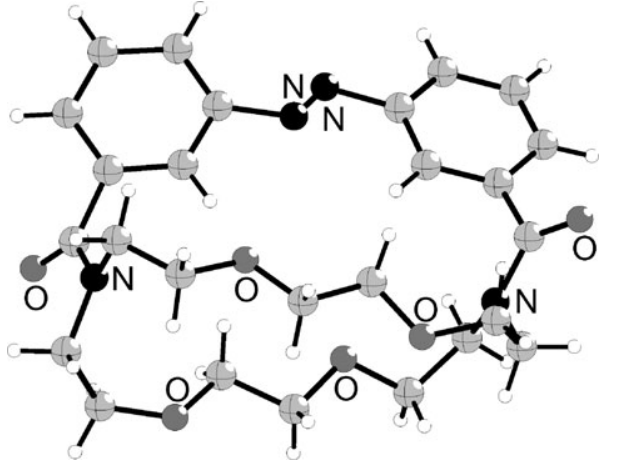

Fig. 1 X-Ray structure of 1.

$10^{-4} \mathrm{M}$ with $\approx 40000$ to 50000 excitation pulses per time step. Five scans taken on different days were averaged to improve the signal-to-noise ratio. Measurements of $\mathbf{2}$ were carried out at a concentration of $1 \times 10^{-3} \mathrm{M}$, resulting in a correspondingly higher signal-to-noise ratio, and two scans were averaged. trans-AB (3), purchased from Merck $(98 \%)$, was measured at a concentration of $7.5 \times 10^{-3} \mathrm{M}$.

\section{Results}

\subsection{UV absorption spectra}

The investigations were initiated by measuring stationary UV/ Vis spectra of the three molecules of interest. All three spectra are broad and unstructured. The weak first absorption maximum of trans-AB (3) is located at $\lambda=445-450 \mathrm{~nm}\left(\mathrm{~S}_{1} \leftarrow \mathrm{S}_{0}\right)$, the strong second transition peaks at $\lambda=319 \mathrm{~nm}\left(\mathrm{~S}_{2} \leftarrow \mathrm{S}_{0}\right)$. ${ }^{24}$ A closer inspection suggests that the $\mathrm{S}_{1} \leftarrow \mathrm{S}_{0}$ absorption maximum of trans-1 may be slightly blue-shifted compared to trans- $\mathrm{AB}(\Delta \lambda \approx 5 \mathrm{~nm})$, while the $\mathrm{S}_{2} \leftarrow \mathrm{S}_{0}$ maximum appears red-shifted by about the same amount. The position of the $\mathrm{S}_{1} \leftarrow \mathrm{S}_{0}$ band maximum of trans-2 is virtually identical with that of trans- $\mathrm{AB}$, while the $\mathrm{S}_{2} \leftarrow \mathrm{S}_{0}$ maximum is slightly red shifted $(\Delta \lambda \leq 3 \mathrm{~nm})$. However, these differences are very minor. Thus, the meta-substitutions on the aromatic rings of the trans-AB nucleus by the aminocarbonyl groups and the crown ether appear to have little influence on the $n \pi^{*}$ and $\pi \pi^{*}$ excited electronic states of the $\mathrm{AB}$ moieties of the molecules. Significant differences between the UV spectra of $\mathbf{1}$ or $\mathbf{2}$ and the parent 3 were only observed at wavelengths of $\lambda \leq 280 \mathrm{~nm}$, where the derivatives exhibit additional absorption bands presumably due to the carbonyl groups, but this difference is not relevant in the present study of the $S_{1}$ state.

\subsection{Time-resolved measurements}

The $\lambda=475 \mathrm{~nm}$ pump pulses in the time-resolved experiments excited the molecules in the red wings of their $S_{1} \leftarrow S_{0}$ absorption bands. Fluorescence was monitored at two detection wavelengths, $\lambda=622$ and $655 \mathrm{~nm}$, close to the peak of the emission spectrum of trans-AB. ${ }^{14-16}$ Since the data taken at the two wavelengths did not show detectable differences they were averaged. The resulting temporal fluorescence profiles for the three molecules are displayed in Fig. 2. Although the data for $\mathbf{1}$ and $\mathbf{2}$ are somewhat noisy due to the poor solubilities and the limited number of experimental runs, it can be seen that the time profiles of the rotation-restricted capped AB $\mathbf{1}$ and the open derivative $\mathbf{2}$ are very similar. Both decay curves are biexponential, with two distinctive time constants. As can be clearly seen, the fluorescence from both derivatives decays more slowly than from the parent compound $\mathbf{3}$.

For a quantitative analysis, the measured time profiles were fitted with a sum of two decaying exponentials convoluted with the IRF using a non-linear least-squares routine based on the Levenberg-Marquardt algorithm. ${ }^{25}$ The two time constants 


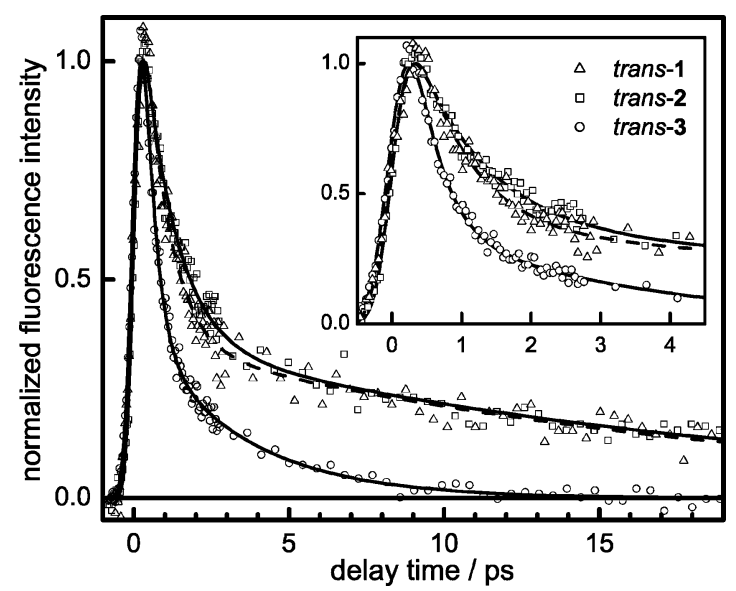

Fig. 2 Measured fluorescence decay curves of the trans-ABs 1, 2, and $\mathbf{3}$ in dioxane. The experimental data are given by the open symbols, the fitted profiles by the solid lines.

and two amplitudes were used as adjustable parameters. In addition, it was checked by varying $\tau_{\text {irf }}$ that the time resolution of the fluorescence measurements agreed with the IRF derived from the Raman signal and the pump-gate cross correlation. The fit results are collected in Table 1. The decay times of the first fluorescence components were found to be

$$
\tau_{1}(\mathbf{1})=0.79 \mathrm{ps}, \tau_{1}(\mathbf{2})=1.05 \mathrm{ps}, \tau_{1}(\mathbf{3})=0.37 \mathrm{ps} ;
$$

the second components were described with

$$
\tau_{2}(\mathbf{1})=20.3 \mathrm{ps}, \tau_{2}(\mathbf{2})=19.0 \mathrm{ps}, \tau_{2}(\mathbf{3})=3.26 \mathrm{ps} .
$$

\section{Discussion}

Considering the detailed molecular mechanisms responsible for the observed dynamics, we start with the following premises: (i) The observed ultrashort fluorescence lifetimes of trans-AB 3 and its derivatives $\mathbf{1}$ and $\mathbf{2}$ studied in the present work are related to ultrafast nonradiative electronic relaxation and transcis isomerization of the molecules. Both processes are assumed to take place through CIs between the $\mathrm{S}_{1}$ and $\mathrm{S}_{0}$ potential energy surfaces (PES) of the molecules near the mid-points of the transcis isomerization reaction coordinates. ${ }^{11,14-21,26,27}$ As has been shown, $\mathrm{S}_{1}$ fluorescence and excited state $\left(\mathrm{S}_{n} \leftarrow \mathrm{S}_{1}\right)$ absorption decay on the same time scale and this decay is accompanied by the appearance of "hot" ground state absorption as signature of the $\mathrm{S}_{1} \rightarrow \mathrm{S}_{0}$ internal conversion. ${ }^{11,12,15,28}$ Thus, nonradiative electronic relaxation and isomerization of $\mathrm{AB}$ are closely connected. (ii) The measured $\mathrm{S}_{1} \rightarrow \mathrm{S}_{0}$ absorption spectra do not indicate significant differences between the respective excited electronic state structures of the three molecules studied. This suggests that the initial states of the molecules after excitation to the $S_{1}$ PES should be comparable. (iii) The observed fast subpicosecond to picosecond fluorescence decay times show that the trans $\rightarrow$ cis isomerization of $\mathrm{AB}$ is a barrierless or nearly barrierless reaction. (iv) The observed two fluorescence time

Table 1 Fluorescence decay times and relative amplitudes for the rotation-restricted capped $\mathrm{AB}$ derivative $\mathbf{1}$, the open derivative $\mathbf{2}$, and the unsubstituted parent compound $\mathbf{3}$ in dioxane solution

\begin{tabular}{lcccc}
\hline trans-AB & $a_{1}(\%)^{a}$ & $\tau_{1} / \mathrm{fs}^{a}$ & $a_{2}(\%)^{a}$ & $\tau_{2} / \mathrm{fs}^{a}$ \\
\hline $\mathbf{1}$ & $75.3(95)$ & $0.79(20)$ & $24.7(56)$ & $20.3(95)$ \\
$\mathbf{2}$ & $72.2(61)$ & $1.05(20)$ & $27.8(51)$ & $19.0(60)$ \\
$\mathbf{3}$ & $79.7(74)$ & $0.37(6)$ & $20.3(39)$ & $3.26(85)$ \\
${ }^{a} 2 \sigma$ standard deviations in parentheses. & & \\
\hline
\end{tabular}

scales $\left(\tau_{1}\right.$ and $\left.\tau_{2}\right)$ are well separated, indicating that they should belong to two genuine dynamical processes.

In the following, we compare the fluorescence decay times of the three ABs of interest, consider work on related stilbenes, and discuss the dynamical processes which may be responsible for the observed two decay time scales.

\subsection{Observed fluorescence decay times}

The striking result of the present work that sheds interesting light on the photoisomerization dynamics of $\mathrm{ABs}$ is that the temporal fluorescence decay profiles of the rotation-restricted capped $\mathrm{AB} \mathbf{1}$ and the chemically similar open AB $\mathbf{2}$ are almost indistinguishable ( $c f$. Fig. 2).

In particular, the fluorescence profiles of all three ABs studied exhibit distinctive biexponential decays. The "fast" fluorescence components $(\approx 75 \%$ rel. amplitude) disappear on the sub-picosecond to picosecond time scale. The respective lifetimes of the derivatives $\mathbf{1}$ and $\mathbf{2}\left(\tau_{1}=0.79\right.$ and $\left.1.05 \mathrm{ps}\right)$ are only about 2.5 times longer than for the parent molecule $\mathbf{3}$ $\left(\tau_{1}=0.37 \mathrm{ps}\right)$. A larger difference (factor of six) between 1 and $\mathbf{2}$ on the one hand and $\mathbf{3}$ on the other was found for the "slow" fluorescence components ( $\approx 25 \%$ rel. amplitude); the observed lifetimes are $\tau_{2} \approx 20$ ps for 1 and 2 vs. $\tau_{2} \approx 3.3$ ps for 3 . Furthermore, the $\tau_{1}$ value for 1 appears to be even slightly shorter than that for $\mathbf{2}$, despite of the extra hindrance of the isomerization due to the crown ether, while the $\tau_{2}$ values are virtually identical. The ratio of the fast and slow fluorescence amplitudes for all three $\mathrm{ABs}$ is $a_{1} / a_{2} \approx 3 / 1$.

We note that it is by no means obvious whether the ultrafast kinetics of a barrierless or near barrierless reaction like the isomerization of $\mathrm{AB}\left(\mathrm{S}_{1}\right)$ should follow a mono- or bi- or multi-exponential decay law. Dynamical models developed for the similar near barrierless excited state isomerization of cis-stilbene (trans-stilbene has a significant potential energy barrier for isomerization and is described by unimolecular rate theory ${ }^{29}$ ) indeed predicted non-exponential decays not attributed to two distinctive dynamical processes. ${ }^{30}$ However, the experimental data on cis-stilbene available at the time showed mono-exponential behaviour. ${ }^{30-32}$ The reported exponential decay time $(\approx 1 \mathrm{ps})$ for cis-stilbene is significantly longer than that for trans- and cis-AB. Further, recent work showed that there is a shallow well on the excited cis-stilbene PES, supporting some low-frequency vibrations. ${ }^{33,34}$ The trans-cis and cistrans isomerization reactions of $\mathrm{AB}\left(\mathrm{S}_{1}\right)$ thus appear to be much better prototypical examples for barrierless reactions than the $c i s$-stilbene reaction.

In agreement with the literature on $\mathrm{AB}$, we take the very clear separation between the observed two decay time scales $\left(\tau_{1}, \tau_{2}\right)$ for $\mathrm{AB}$ and especially for the $\mathrm{AB}$ derivatives as evidence for (at least) two distinctive dynamical processes. ${ }^{14,15,21,26,27}$ The striking similarities then strongly suggest that photoisomerization of the $A B$ derivatives $\mathbf{1}$ and $\mathbf{2}$ is governed by the same molecular mechanisms. Whether or to which extent this also applies for the unsubstituted parent $\mathrm{AB} 3$ remains a question which we defer to the end of this discussion. The comparison of the experimental decay times for the parent $\mathrm{AB}$ on the one hand and the derivatives on the other hand suggests that there are at least subtle differences.

\subsection{Comparison with related stilbenes}

As mentioned, the longstanding question is whether the reaction coordinate for the photoisomerization of $\mathrm{AB}$ is better characterized as "rotation" about the central NN bond or "inversion" at one (or both) of the $\mathrm{N}$ atoms. While the actual pathways may be more complex, a fundamental classification of the dynamics along these two lines is justified by comparison of the photoisomerization quantum yields of the cited capped $\mathrm{ABs}^{9,10}$ (e.g., 1) and similar capped stilbenes. In particular, 
Rau and Waldner recently reported that the photoisomerization quantum yield for a trans-stilbenophane is $\Phi=0 .{ }^{35}$ This highly intriguing result is rationalized by the blocking of the "rotational" reaction coordinate in the stilbenophane.

For the case of stilbene and for several polyenes, it is well known that the cis-trans isomerization coordinate is much more complex than a simple large-amplitude rotation. ${ }^{33,34}$ The actual isomerization coordinate for stilbene is assumed to be a mixture of several internal coordinates showing torsional motion around the ethylenic $\mathrm{CC}$ bond, including out-of-plane $\mathrm{CH}$ wag and other oscillatory motions on the multidimensional PES, which minimize solvent friction. ${ }^{30,31}$ The term "hulatwist" motion has been coined to describe the combined effect. ${ }^{33,34}$ However, the photoisomerization quantum yield of $\Phi=0$ for the trans-stilbenophane shows that under the experimental conditions used neither rotation nor hula-twist nor inversion nor another type of motion appears to be a feasible reaction coordinate for that molecule. ${ }^{35}$

With these premises, the contrast between the photoisomerization quantum yield of $\Phi=0$ for the stilbenophane ${ }^{35}$ compared with the "normal" quantum yields found for the similar rotation-restricted azobenzophanes and the crown ether capped AB $1(\Phi \approx 0.24 \text { and } 0.29)^{10}$ can be rationalized only if the capped ABs undergo photoisomerization by "inversion". The same route is thus suggested for the open AB derivative 2 . The inversion may not take place via the originally proposed semi-linear CNNC configuration, for which there appears to be a potential energy barrier. ${ }^{17,18}$ Instead, Diau proposed a concerted inversion pathway, via a CI between the $\mathrm{S}_{1}$ and $\mathrm{S}_{0}$ states at an almost linear CNNC configuration. ${ }^{17} \mathrm{It}$ is of interest in this context that according to a picosecond Raman study the NN bond of trans-AB in the $\mathrm{S}_{1}$ state keeps a double bond character. ${ }^{36}$ In addition, we would like to mention the possibility of a "hula-twist" reaction coordinate similar to the stilbene case, involving some out-of-plane motions of the $\mathrm{N}$ atoms as a "non-planar inversion" variant. The hula-twist motion, which has not yet been theoretically explored in sufficient detail, would lead to the same orientation of the phenyl ring as a planar inversion, whereas rotation of the phenyl group about the $\mathrm{NN}$ bond exchanges the $\mathrm{C}$ atoms on the two sides of the ring.

\subsection{Mechanistic explanation of the bi-exponential decay profiles}

Having identified inversion as the reaction pathway for the AB derivatives $\mathbf{1}$ and $\mathbf{2}$, the origin of the biexponential decay curves moves into focus. Here, possible differences have to be taken into account between the dynamics of unsubstituted $\mathrm{AB}^{15,21,27}$ and the $\mathrm{AB}$ derivatives. We shall discuss common features first and specific differences afterwards.

The starting point for the dynamics is defined by the location of the initial wavepacket prepared on the $S_{1}$ PES. Considering the $n \pi^{*}$ nature of the $S_{1} \leftarrow S_{0}$ transition, where a non-bonding electron is removed from one of the $\mathrm{N}$ atom lone pairs, the Franck-Condon region is determined by a high degree of excitation of the $\mathrm{S}_{1} \mathrm{NNC}$ bending vibration. Incidentally, this vibration coincides basically with the inversion reaction coordinate. In addition, there is a shortening of the $\mathrm{CN}$ bond in the $\mathrm{S}_{1}$ state compared to the $\mathrm{S}_{0}$ state. The Franck-Condon determined initial location of the excited wavepackets on the $\mathrm{S}_{1}$ PES at $t=0$ should be similar for all three ABs.

In line with previous arguments, ${ }^{15,21,27}$ the fast initial decay times $\left(0.3 \leq \tau_{1} \leq 1 \mathrm{ps}\right.$, respectively) are therefore attributed to fast motion of the excited wavepackets on the $\mathrm{S}_{1}$ PES away from the Franck-Condon region towards the CI (or CIs) with the $\mathrm{S}_{0}$ PES. The direct motion leads the wavepacket along the steepest descent on the $S_{1}$ PES. The even faster $\left(\tau_{1} \leq 0.1 \mathrm{ps}\right)$ decay of cis-compared to trans-AB is thus explained by a steeper gradient of the $S_{1}$ PES near the cis configuration. ${ }^{15,16}$
At least initially, the wavepacket should move mainly along the NNC bending coordinate. This conclusion has been supported by a recent semiclassical dynamics calculation. ${ }^{20}$ The longer $\tau_{1}$ values of $\mathbf{1}$ and $\mathbf{2}$ compared to the parent molecule $\mathbf{3}$ are attributed to increased solvent friction due to the extended side chains. In addition, the direct wavepacket motion in the direction of steepest descent on the $S_{1}$ PES may be accompanied by some rapid partial intramolecular vibrational redistribution (IVR) among vibrational modes which are strongly coupled with the NNC bending mode. The molecules were prepared with about $2000-3000 \mathrm{~cm}^{-1}$ of internal energy above the $S_{1}$ origin, corresponding to several vibrational quanta which may be partially redistributed.

The slow fluorescence decay times of $\tau_{2} \approx 20$ ps for the AB derivatives are attributed to their transition to the $\mathrm{S}_{0}$ state through the $\mathrm{CI}$ along the inversion coordinate. This dynamics can be pictured as indirect, "diffusive" motion on the $S_{1}$ PES. The molecules may execute a sort of "meandering" motion, miss the CI on their first pass, and survive on the $\mathrm{S}_{1}$ PES until they find the CI from different directions at a later time. The diffusive motion is partially a consequence of the fact that the CI is not located exactly on the initial direct path of the wavepacket and partially a consequence (and manifestation) of fast partial "IVR". Following Diau, ${ }^{17}$ the CI between the $S_{1}$ and $\mathrm{S}_{0}$ states is located along the concerted inversion coordinate at an almost linear CNNC configuration. The funneling of the wavepacket through the $\mathrm{CI}$ and the subsequent wavepacket evolution on the $\mathrm{S}_{0}$ PES determine whether the product molecules end up in the cis or the trans configurations. The higher number of vibrational degrees of freedom and the increased solvent friction of the AB derivatives lead to a much slower "diffusive" arrival of the excited wavepacket at the $\mathrm{CI}$, hence the increase of $\tau_{2}$ from $\approx 3.3$ ps to $\approx 20$ ps with increasing molecular size. Additionally, on the time scale of $\approx 20$ ps the molecules in the $S_{1}$ state experience substantial vibrational relaxation by the solvent. Thus, the $\tau_{2}$ values for the derivatives are essentially attributed to vibrationally relaxed $S_{1}$ molecules. For the unsubstituted $A B$, $\tau_{2}$ is too short for a large degree of vibrational relaxation within the $S_{1}$ state. Eventually, since the current experiments had to be optimized for parallel polarization detection to observe the extremely weak fluorescence from $\mathbf{1}$ and $\mathbf{2}$, it cannot be excluded that rotational depolarization may play a small role.

Considering the unsubstituted parent trans- or cis-AB, the "inversion" mechanism has received support from Nägele et al. and Satzger et al., ${ }^{1,15,27}$ who compared time-resolved absorption results on trans- and cis-AB after $\mathrm{S}_{1}\left(\mathrm{n} \pi^{*}\right)$ and after $\mathrm{S}_{2}$ $\left(\pi \pi^{*}\right)$ excitation. Transient signals of trans-AB in ethanol or DMSO in the case of $\mathrm{S}_{1}$ excitation were found to exhibit decay times of $\tau_{1} \approx 0.3$ and $\tau_{2} \approx 2-3 \mathrm{ps}$; those of cis-AB could be described with decay times of $\tau_{1} \approx 0.1-0.17$ and $\tau_{2} \approx 0.9-1.0$ ps, in excellent agreement with the fluorescence up-conversion data on the $\mathrm{S}_{1}$ state obtained in our laboratory ${ }^{16}$ and by $\mathrm{Lu}$ et al. ${ }^{14}$ Apart from an additional very fast decay time $(\lesssim 0.1 \mathrm{ps})$ attributed to internal conversion from the $S_{2}$ to the $S_{1}$ state, virtually the same decay times were found following $S_{2}$ excitation, in agreement in this case with fluorescence up-conversion data by Fujino et al. ${ }^{37}$ These results were interpreted by Satzger et al. in terms of a two step model, whereby an ultrafast radiationless transition ( $\lesssim 0.1$ ps) takes the molecules first from $S_{2}$ to $S_{1}$. The actual isomerization then follows from the subsequent large-amplitude motions on the $\mathrm{S}_{1}$ PES towards the CI with the $\mathrm{S}_{0}$ state close to the midpoint of the cis-trans isomerization coordinate. Further slow processes appearing on the 5-20 ps time scale were attributed to vibrational cooling of the produced hot ground state molecules. Likewise, Lednev et al., ${ }^{13}$ who reported transient absorption measurements of $\mathbf{1}$ after excitation to the $S_{2}$ state, found an exponential decay with 
a lifetime of $\tau=2.6 \mathrm{ps}$, somewhat longer than the value of $\tau_{1}$ found in the present work. In their case, the molecules were concluded to experience a fast internal conversion to the $S_{1}$ state within $<0.5$ ps. The differences between $\mathrm{S}_{2}$ vs. $\mathrm{S}_{1}$ excitation thus reflect the different starting positions of the dynamics of the wavepackets on the $\mathrm{S}_{1}$ PES

However, recent femtosecond fluorescence depolarization measurements of trans- $\mathrm{AB}$ excited to the $\mathrm{S}_{1}$ state in solvents of different viscosity by Chang et al. showed that the dynamics of the prototypical parent $\mathrm{AB}$ is considerably more complex. ${ }^{21}$ They observed that the fluorescence anisotropy of trans-AB in hexane decays with the same time constant as the slow fluorescence $\left(\tau_{2}\right)$. In contrast, the fluorescence anisotropy in ethylene glycol did not show a discernable decay. They concluded that both the rotation and the inversion routes are important, but that their relative contributions depend on the conditions. In low viscosity solvents (hexane) they assumed isomerization predominantly via out-of-plane CNNC torsional motion and the CI at the twisted configuration predicted by theoretical work, ${ }^{17-20}$ while the concerted in-plane inversion pathway ${ }^{17}$ was preferred in high-viscosity solvents (ethylene glycol). Quoted differences between the decay times and photoisomerization quantum yields of trans- vs. cis- $\mathrm{AB}$ and $\mathrm{S}_{2} v s . \mathrm{S}_{1}$ excitation thus reflect different starting points of the excited wavepackets on the $S_{1}$ PES, different relative contributions of the rotation and inversion reaction pathways, and different branchings of the wavepackets into the cis or trans directions at the $\mathrm{S}_{1}-\mathrm{S}_{0}$ CIs.

\section{Conclusions}

In conclusion, we have studied the temporal fluorescence decay profiles of a rotation-restricted trans-AB derivative capped by a crown ether (1), a chemically similar open derivative (2), and the unsubstituted parent trans-AB (3) following excitation to the $S_{1}\left(n \pi^{*}\right)$ state. The observed biexponential fluorescence profiles for $\mathbf{1}$ and $\mathbf{2}$ were almost indistinguishable within experimental error, suggesting strongly that trans-cis isomerization of $\mathbf{1}$ and $\mathbf{2}$ after $\mathrm{S}_{1}$ excitation is governed by the same mechanism. The results support the idea that the internal conversion and isomerization of the molecules proceeds through a conical intersection between the $\mathrm{S}_{1}$ and $\mathrm{S}_{0}$ states. The very fast first fluorescence decay time is attributed to the initial direct motion of the excited wavepacket on the $\mathrm{S}_{1}$ PES away from the Franck-Condon region. The slower second fluorescence decay time is assigned to the transition from to the $S_{0}$ to the $S_{1}$ state by a slower, "diffusive" motion. The isomerization of the capped AB 1 and the open derivative $\mathbf{2}$ is concluded to proceed via a planar (and probably concerted) "inversion" at the $\mathrm{N}$ atom(s) or a type of "hulatwist" motion of the $\mathrm{N}$ atom(s) and phenyl ring(s). Further theoretical studies are needed to fully characterize the possible inversion routes.

\section{Acknowledgements}

The financial support of this work by the Deutsche Forschungsgemeinschaft and the Fonds der Chemischen Industrie is gratefully acknowledged. A.K. thanks the Fonds der Chemischen Industrie for a Liebig stipend, T.P. thanks the Fonds der Chemischen Industrie for a PhD fellowship.

\section{References}

1 T. Ikeda and O. Tsutsumi, Science, 1995, 268, 1873.

2 Z. F. Liu, K. Hashimoto and A. Fujishima, Nature, 1990, 347, 658 .

3 I. Willner and S. Rubin, Angew. Chem., Int. Ed., 1996, 35, 367.

4 T. Hugel, N. B. Holland, A. Cattani, L. Moroder, M. Seitz and H. E. Gaub, Science, 2002, 296, 1103 .

5 N. B. Holland, T. Hugel, G. Neuert, A. Cattani-Scholz, C. Renner, D. Oesterhelt, L. Moroder, M. Seitz and H. E. Gaub, Macromolecules, 2003, 36, 2015.

6 L. Ulysse, J. Cubillos and J. Chmielewski, J. Am. Chem. Soc., 1995, 117, 8466.

7 J. R. Kumita, O. S. Smart and G. A. Woolley, Proc. Natl. Acad. Sci. USA, 2000, 97, 3803.

8 S. Spörlein, H. Carstens, H. Satzger, C. Renner, R. Behrendt, L. Moroder, P. Tavan, W. Zinth and J. Wachtveitl, Proc. Natl. Acad. Sci. USA, 2002, 99, 7998.

9 H. Rau and E. Lüddecke, J. Am. Chem. Soc., 1982, 104, 1616.

10 H. Rau, J. Photochem., 1984, 26, 221.

11 T. Nägele, R. Hoche, W. Zinth and J. Wachtveitl, Chem. Phys. Lett., 1997, 272, 489.

12 I. K. Lednev, T.-Q. Ye, P. Matousek, M. Towrie, P. Foggi, F. V. R. Neuwahl, S. Umapathy, R. E. Hester and J. N. Moore, Chem. Phys. Lett., 1998, 290, 68.

13 I. K. Lednev, T.-Q. Ye, L. C. Abbott, R. E. Hester and J. N. Moore, J. Phys. Chem. A, 1998, 102, 9161.

14 Y.-C. Lu, C.-W. Chang and E. W.-G. Diau, J. Chin. Chem. Soc., 2002, 49, 693.

15 H. Satzger, S. Spörlein, C. Root, J. Wachtveitl, W. Zinth and P. Gilch, Chem. Phys. Lett., 2003, 372, 216.

16 T. Pancur, PhD thesis, Christian-Albrechts-Universität zu Kiel, 2004.

17 E. W.-G. Diau, J. Phys. Chem. A, 2004, 108, 950.

18 A. Cembran, F. Bernardi, M. Garavelli, L. Gagliardi and G. Orlandi, J. Am. Chem. Soc., 2004, 126, 3234.

19 T. Ishikawa, T. Noro and T. Shoda, J. Chem. Phys., 2001, 115, 7503.

20 C. Ciminelli, G. Granucci and M. Perisco, Chem. Eur. J., 2004, 2327.

21 C.-W. Chang, Y.-C. Lu, T.-T. Wang and E. W.-G. Diau, J. Am. Chem. Soc., 2004, 126, 10109.

22 T. Wilhelm, J. Piel and E. Riedle, Opt. Lett., 1997, 22, 1494.

23 S. Shinkai, T. Nakaji, Y. Nishida, T. Ogawa and O. Manabe, J. Am. Chem. Soc., 1980, 102, 5860 .

24 H.-H. Perkampus, UV-Vis Atlas of Organic Compounds, VCH, Weinheim, 2nd edn., 1992, vol. 1.

25 W. H. Press, B. P. Flannery, S. A. Teukolsky and W. T. Vetterling, Numerical Recipes in C, Cambridge University Press, Cambridge, 1988.

26 T. Schultz, J. Quenneville, B. Levine, A. Toniolo, T. J. Martinez, S. Lochbrunner, M. Schmitt, J. P. Shaffer, M. Z. Zgierski and A. Stolow, J. Am. Chem. Soc., 2003, 125, 8098.

27 H. Satzger, C. Root and M. Braun, J. Phys. Chem. A, 2004, 108, 6265 .

28 P. Hamm, S. Ohline and W. Zinth, J. Chem. Phys., 1997, 106, 519.

29 J. Schroeder, T. Steinel and J. Troe, J. Phys. Chem. A, 2002, 106, 5510 .

30 D. C. Todd, J. M. Jean, S. J. Rosenthal, A. J. Ruggiero, D. Yang and G. R. Fleming, J. Chem. Phys., 1990, 93, 8658.

31 A. Abrash, S. Repinec and R. M. Hochstrasser, J. Chem. Phys., 1990, 93, 1041 .

32 L. Nikowa, D. Schwarzer, J. Troe and J. Schroeder, J. Chem. Phys., 1992, 97, 4827.

33 K. Ishii, S. Takeuchi and T. Tahara, Chem. Phys. Lett., 2004, 398, 400.

34 W. Fuß, C. Kosmidis, W. E. Schmid and S. A. Trushin, Chem. Phys. Lett., 2004, 385, 423.

35 H. Rau and I. Waldner, Phys. Chem. Chem. Phys., 2002, 4, 1776

36 T. Fujino and T. Tahara, J. Phys. Chem. A, 2000, 104, 4203.

37 T. Fujino, S. Y. Arzhantsev and T. Tahara, J. Phys. Chem. A, 2001, 105, 8123 . 